\title{
INTELLIGENT FURNACE TECHNOLOGY FOR ANNEALING AND GALVANIZING LINES *
}

\author{
Ulrich Sommers ${ }^{1}$ \\ Carsten Andreas Klein ${ }^{2}$ \\ Fritz Brüh ${ }^{\beta}$ \\ Caesar Sasse $^{4}$
}

\begin{abstract}
The Intelligent Furnace (I-Furnace) by SMS group is a smart furnace process and production optimization model. The combination of various tools leads to an optimized heat treatment and production process. It combines a mathematical/physical model to control the furnace with EMG's IMPOC system for online strength measurement and a newly developed annealing microstructure model to predict material properties. This paper reports about the revamping of an existing galvanizing line, where a mathematical/physical control model has been implemented in combination with an online quality measurement system. This way, the process improvement of continuous galvanizing lines and the quality management of a range of steels including AHSS will be demonstrated. Furthermore, it gives information about the development of the annealing microstructure model to predict material characteristics. Eventually, the I-Furnace will be presented according to the current development and reference status.
\end{abstract}

Keywords: FURNACE; ANNEALING; GALVANIZING; MICROSTRUCTURE; AUTOMOTIVE GRADES; PROCESSING LINES; AHSS.

1 Dr.; Deputy General Manager R\&D - RDZS; Technology Processing Lines/ Furnace; SMS group; Düsseldorf, Germany

2 Dipl.-Phys.; Head of Fundamentals and Models Strip Processing Lines/ Furnaces, Heat Technology R\&D - RDZ SMS group GmbH, Düsseldorf, Germany

3 Dr.-Ing. Metallurgy; Executive Vice President, Strip Processing Lines and Furnace Technology, SMS group, Düsseldorf, Germany.

4 Technical Communications M.A., Technical Marketing Manager, Strip Processing Lines and Furnace Technology, SMS group, Hilden, Germany. 


\section{INTRODUTION: CHALLENGES IN THE PRODUCTION OF ADVANCED HIGH-STRENGTH STEELS}

The final material properties and the final product quality of advanced high-strength steel grades (AHSS grades) are determined by heating and cooling in continuous annealing or galvanizing lines according to the appropriate annealing curve. Today's progress in the flat sheet industry is mainly driven by the significantly growing share of AHSS grades. In addition, high demands from the automotive market lead to increased constraints for steel makers. Without a doubt, one key element is the product quality, which is mandatory for automotive materials.

There are several challenges for steelmakers concerning the production of modern AHSS grades. Flexible process conditions are required to continuously adapt the lines to the market requirements. The steel compositions of modern high strength grades are modified, which makes changes in the metallurgical and finishing process necessary. The strip temperature has to obey the given limits to achieve the high product quality. Additionally, a stronger consideration of the upstream processes is necessary.

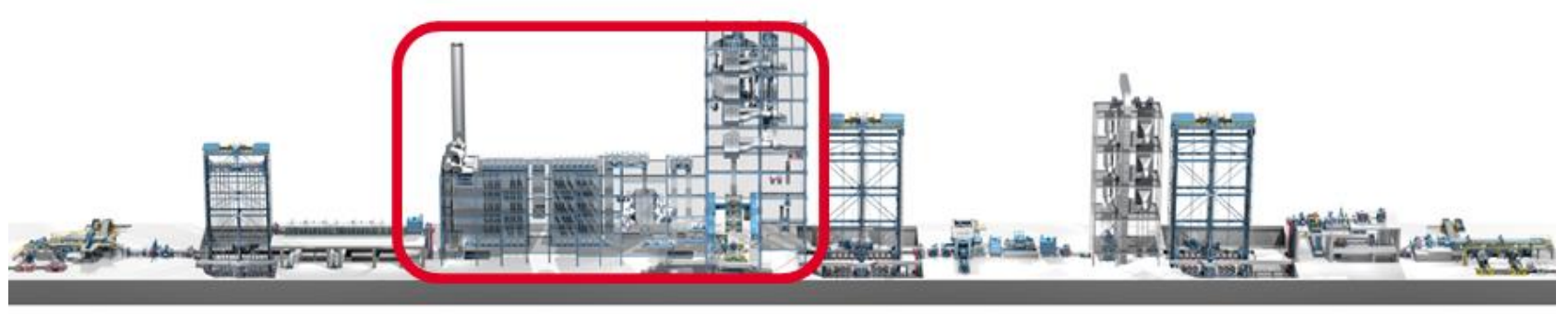

Fig.1 - The annealing process determines the material properties and final product quality in cold-strip processing lines

\section{I-FURNACE}

The I-Furnace (Intelligent Furnace) is a smart furnace process and production optimization model newly developed by SMS group, Germany. The combination of various tools leads to an optimized heat treatment and production process. It combines furnace control, online strength measurement and a model to predict the material properties after the treatment. This intelligent combination of efficient tools is a great step forward towards an autonomously working furnace.

There are several advantages for the customer resulting from the use of this system:

- A capacity increase up to 15 percent is possible due to a better utilization of the furnace capacity as well as an efficient production planning and transition behavior

- Energy savings can be realized due to a better temperature control close to its lower limit

- Special sophisticated grades can be produced more easily

- Improved material characteristics - especially for coils with deviations

- Increased yield of high-quality material 


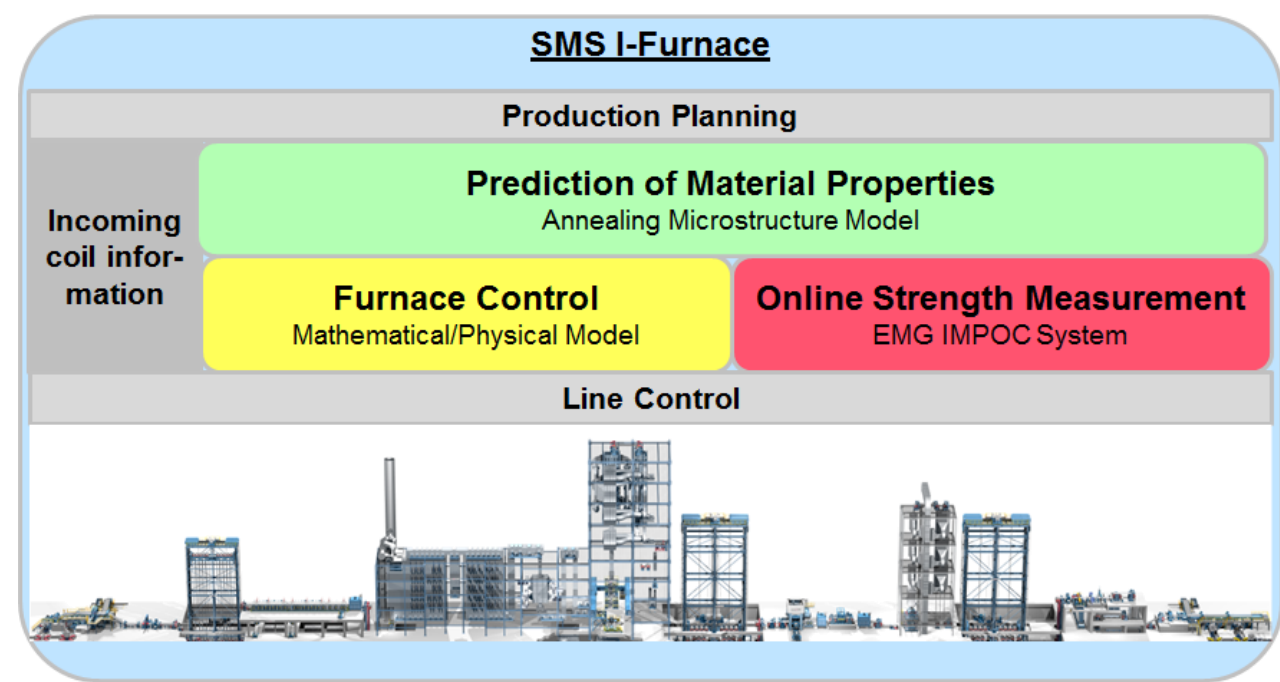

Fig.2 - A powerful combination of three tools leads to optimized coil heat treatment

To control the furnace and the heat treatment process an advanced mathematical/physical model by Drever International, Belgium, is used. Drever, a company of SMS group, supplies a full range of furnace technology for steel strip and is the leading supplier of furnaces for galvanizing and annealing lines of carbon steel strips.

For a continuous online check of material properties the IMPOC system of EMG Automation GmbH, Germany, is integrated in the I-Furnace concept. EMG Automation $\mathrm{GmbH}$, a company of the elexis group, belongs to the leading suppliers of quality assurance and strip guiding systems for fast running continuous production processes in the metal and especially in the steel industry.

Last but not least the newly developed annealing microstructure model (AMM) by SMS group is incorporated to predict the material properties according to the respective heat treatment and production parameters. The SMS group is a group of companies internationally active in plant construction and mechanical engineering for the steel and nonferrous metals industry.

\section{Online strength measurements - EMG IMPOC}

The IMPOC measuring system integrated in cold strip processing lines continually checks the material's tensile strength and yield strength during production. This allows to monitor the behavior of the strip over the entire strip length and width. There are further capabilities in the IMPOC system, like the setting up of material limits, which are also used for coil release during the production. Furthermore, the IMPOC system gives an automatic alarm when thresholds are exceeded. Since today some automotive manufacturers use this system for checking materials, the material requirements of the automotive industry are increasing and forcing the steelmakers to check the quality within their lines by themselves before delivering to the customers.

The IMPOC system is perfect for automatic, non-destructive, online testing of ferromagnetic steel strips with thicknesses from 0.15 to 6 millimeters. This system is based on two identically constructed sensors, which are arranged on the top and bottom surface of the strip. Each sensor consists of a magnetizing coil and a magnetic field probe for recording the magnetic remanence. Tensile and yield strength are subsequently calculated by multiple regression on the measured values from destructive material testing. 


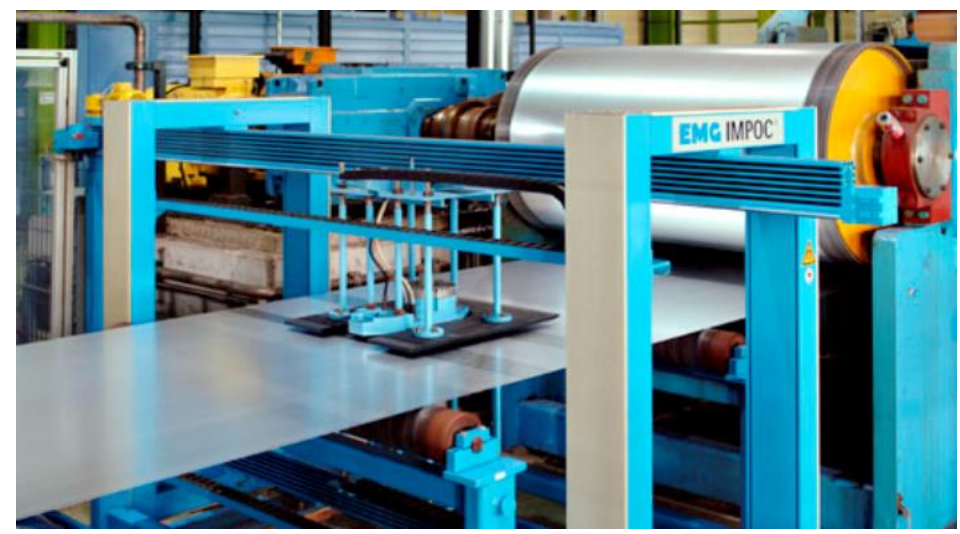

Fig.3 - EMG IMPOC system for online strength measurement in a continuous galvanizing line

\section{Furnace control - mathematical/physical furnace model}

At production level, the operators in cold strip processing lines face a high variability of parameters which need to be integrated in the thermal process: steel type, strip dimension, heat cycle, cooling rate, target speed, etc. These factors are taken into consideration to create a production schedule including constraints, which in turn induce parameter changes that need to be managed during production.

The mathematical/physical model responds to these day-by-day situations with an advanced transition management system. It allows full automatic control of the furnace, and also of the process line speed. For each scheduled coil, setpoints are calculated, which lead coil after coil to recurring changes of the furnace working points and thus the strip temperature profile. Cascading of transitions as well as collision of transitions are common among producers and are managed within the model. The advanced transition management integrates the dynamic behavior of the furnace for an optimized control. The process behavior is forecast within the transition calculation, which traces the optimum control of the furnace and line speed. The transition results are displayed online to guarantee operational transparency. A maximum production rate at the same time as high quality is the core objective of the control concept.

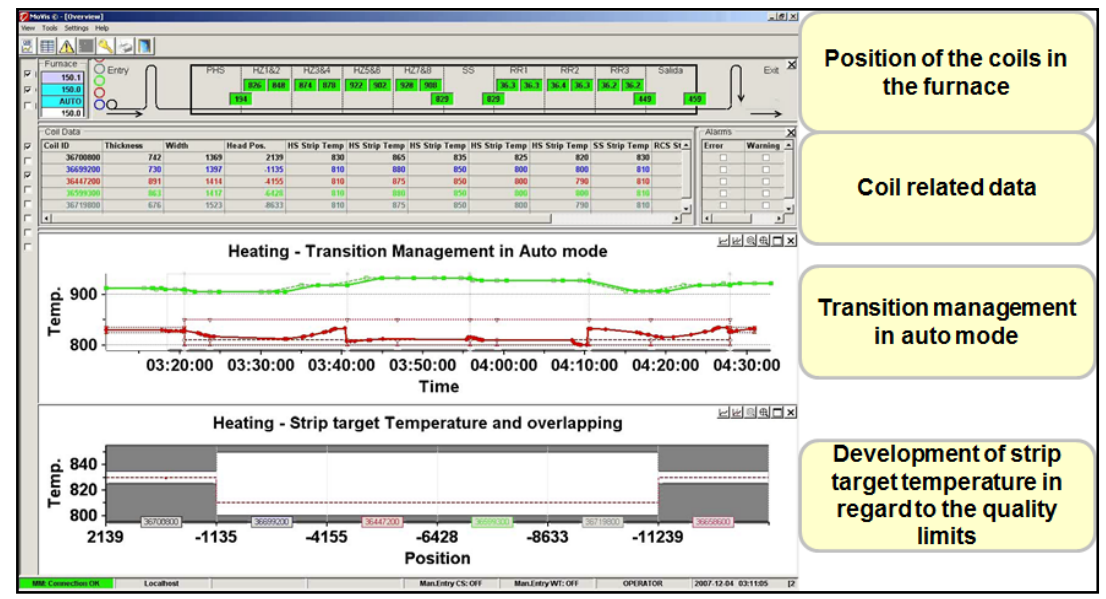

Fig.4 - Automatic furnace and line speed control by furnace mathematical/physical model

\section{Predication of material properties - annealing microstructure model}

The newly developed annealing microstructure model allows the prediction of the material properties after the annealing process. The simulation of the properties can take place with two approaches: On the one hand a data driven model can be used online and on the other hand a metallurgical model can be used offline. 
The data driven model can be used online during production. Based on various measurements and evaluations the preset values can be optimized with numerical methods for each coil. Input parameters are both data which are related to the plant or the coil (plant data, product data, and process data collected during the upstream process) and data which are measured online during the process. Furthermore, the calculations are based on a comprehensive knowledge of the process. The optimization based on these parameters result in more consistent properties for yield and tensile strength.

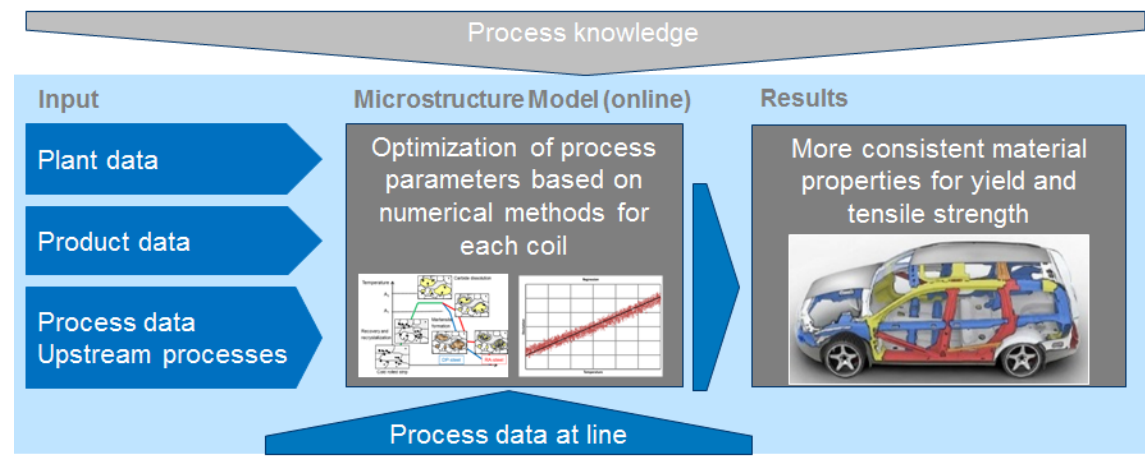

Fig.5 - Description of the data driven online Annealing Microstructure Model

The metallurgical model has been developed for an offline prediction. It simulates the mechanical properties (tensile and yield strength) according to the development of the microstructure. The physical calculation of the microstructure composition is based on heating cycle and process parameters, which can be optimized. This simulation gives also indications about the limits of existing annealing furnaces concerning the production of new products. Thus, this model can be used to design the process and for research activities. Another beneficial use is the model based substitution of expensive alloying elements.

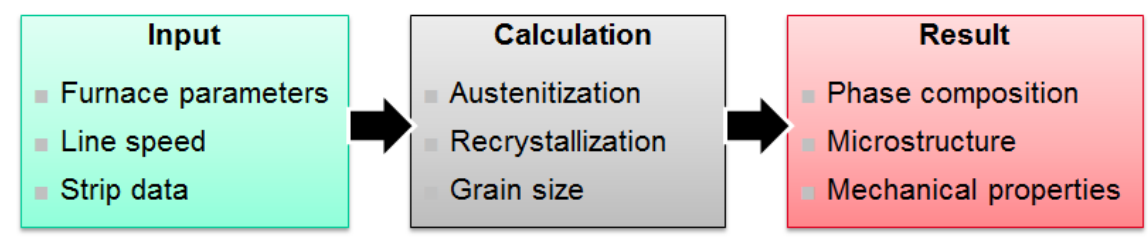

Fig.6 - Description of the metallurgical offline Annealing Microstructure Model

\section{SEGAL - IMPLEMENTATION OF FURNACE CONTROL AND ONLINE STRENGTH MEASUREMENT}

\subsection{Revamp of an automotive line}

The continuous galvanizing line of Segal (Tata Steel), Belgium, was built in the mid-eighties as the first line in Europe designed specifically for automotive products. At this time direct fired furnaces combined with a radiant tube section were common, but Segal relied on full radiant tube technology from Drever as a breakthrough. This dedicated production line called for different sections in the annealing process, nowadays commonly found on high-end production units. The full radiant tube furnace is followed by a slow cooling section, then a rapid cooling section and later on a long galvannealing section.

The Segal line has always been regarded as state of the art technology, also for the next generation of high strength steels. Continuous investments led to the introduction of up-to-date process equipment. On the annealing process, there is a new high hydrogen management in the rapid cooling section, a new reheating section, and very recently a new high performance after pot cooling section.

In the process of continuous improvement, the furnace mathematical/physical model was revamped in 2014. The model now integrates the latest self-adaptation technique, advanced transition 
management technology and annealing process optimization. It is designed to allow a synchronous control of the annealing furnace and the process line speed. Along the new mathematical model implementation, an online quality measurement device has been installed, the EMG IMPOC.

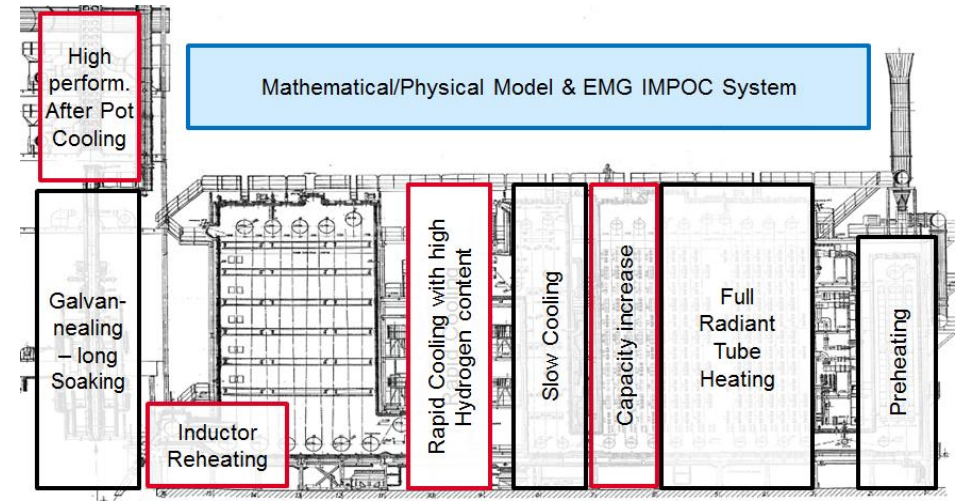

Fig.7 - Accompanied by some other revamp activities a new mathematical/physical model and an EMG IMPOC system have been implemented in the furnace of Segal's continuous galvanizing line

\subsection{Process optimization: mathematical/physical model}

Over the lifespan of a line, the product mix steadily shifts from relatively simple steels to increasingly complex grades. This shift follows from the response to higher customer demands, the increase of the market share of AHSS grades and the introduction of the next generation of steel grades. Due to the changing portfolio, galvanizers face a growing variability of parameters in their production schedule. The annealing furnace at Segal was already driven by a mathematical model. The increasing complexities of the process and the high requirement in quality have led to the introduction of the newest mathematical/physical model.

The mathematical/physical model for furnace control with advanced transition management technology is designed to aim for maximum productivity, while managing the quality of the products. Therefore it helps to save energy, increase the yield and quality and it simplifies the operation.

The most significant result due to the use of the model is an increased line speed. During a production campaign of eleven wide coils (up to $1,760 \mathrm{~mm}$ ) speed increases between 13.5 and 15.1 percent could be realized. This means that for every coil the processing time could be remarkably reduced which leads to a vast capacity increase of the line.

\begin{tabular}{|c|c|c|c|}
\hline Coil & $\begin{array}{c}\text { Speed } \\
\text { Scheduled } \\
{[\mathrm{m} / \mathrm{min}]}\end{array}$ & $\begin{array}{c}\text { Speed } \\
\text { Driven } \\
{[\mathrm{m} / \mathrm{min}]}\end{array}$ & $\begin{array}{c}\text { Ratio } \\
{[\%]}\end{array}$ \\
\hline 240QDY & 79 & 90 & 13.9 \\
\hline $240 Q B I$ & 65 & 74 & 13.8 \\
\hline 240QBE & 65 & 74 & 13.8 \\
\hline $240 Q B H$ & 65 & 74 & 13.8 \\
\hline $240 Q B M$ & 73 & 84 & 15.1 \\
\hline $240 Q B N$ & 73 & 84 & 15.1 \\
\hline $240 P 98$ & 79 & 90 & 13.9 \\
\hline $240 \mathrm{Pg} 9$ & 79 & 90 & 13.9 \\
\hline 240QBG & 79 & 90 & 13.9 \\
\hline $240 Q B F$ & 89 & 101 & 13.5 \\
\hline $240 Q B 2$ & 89 & 101 & 13.5 \\
\hline
\end{tabular}

Fig.8 - Production campaign of wide coils with scheduled speed, driven speed and the speed increase in percent due to the use of the newest mathematical/physical model with advanced transition management 


\subsection{Quality improvement: online strength measurement}

Due to the consideration of the data collected by the online strength measurement system EMG IMPOC even significant quality improvement could be demonstrated. One example for the quality improvement due to online strength measurement has been the elimination of strength deviations within the length of some coils. The most likely reason for this behavior are the cooling conditions after hot rolling, but since the deviations showed a systematic behavior they could be compensated within the furnace. This way the quality of the final product could be increased significantly due to the consideration of both the EMG IMPOC system and the mathematical/physical model.

The changes in mechanical properties over the coil length showed a systematic behavior for some coils which resulted in a typical "camel" shape. In order to improve the quality of the steel parameters have been evaluated that can be changed fairly quickly and can change the IMPOC value. From the metallurgical analysis and the linear models, the temperature after slow cooling was identified as a good way to improve the quality of the steel. Changing the slow cooling strip temperature is better than changing the annealing temperature, because it is possible to change it more quickly.

By using the temperature at the exit of the slow cooling it is possible to compensate the "camel" shape profile of the DP 600 coils. The higher temperature after slow cooling will cause that relatively more austenite is retained before rapid cooling. This austenite will transform into martensite. Thus the higher temperatures lead to a higher martensite fraction, which gives a higher tensile strength. During some trials this behavior could be verified and on optimized annealing curve for this kind of coils could be found.
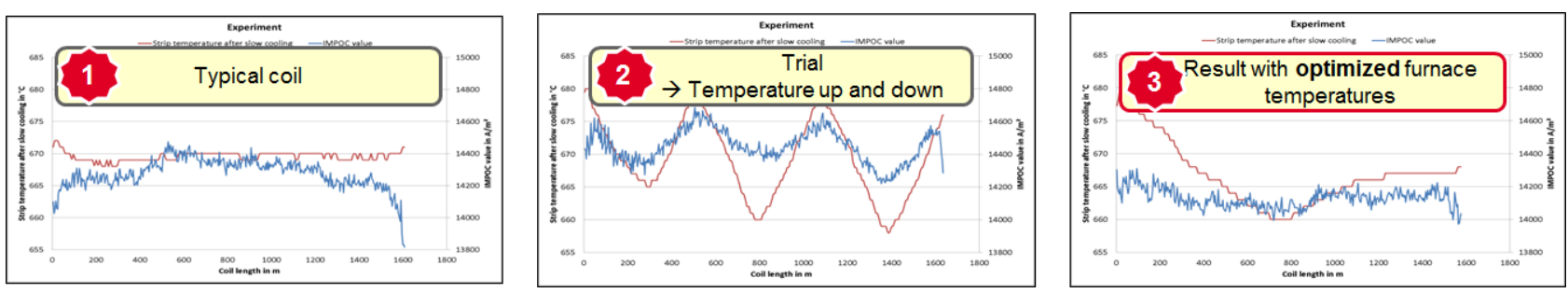

Fig.9 - From left to right: The typical coil shows a "camel" shape curve of the IMPOC value in blue and the strip temperature at the exit of the slow cooling in red, via several trials the effect of a changed slow cooling temperature was tested, and eventually the slow cooling temperature has been optimized and thus the "camel" shape has been eliminated

\subsection{Quality improvement: consideration of upstream data}

A more comprehensive quality improvement is possible with a consideration of the upstream data, since the quality deviations are often influenced or caused by upstream parameters. One example has been the modeling of steel quality for DP 800 based on measurements taken at the hot strip mill and the galvanizing line.

When using the data from the hot strip mill (e.g. slab temperature going into the rougher, slab temperature going into the finishing mill and coiling temperature) and the galvanizing line (e.g. strip temperature after heating, strip temperature after soaking, strip temperature after slow cooling, line speed and slow cooling time) the quality of the produced galvanized cold strip could be predicted well. Of course, a precise knowledge of the resulting strength distribution after the heat treatment allows more efficient compensations of possible deviations. 


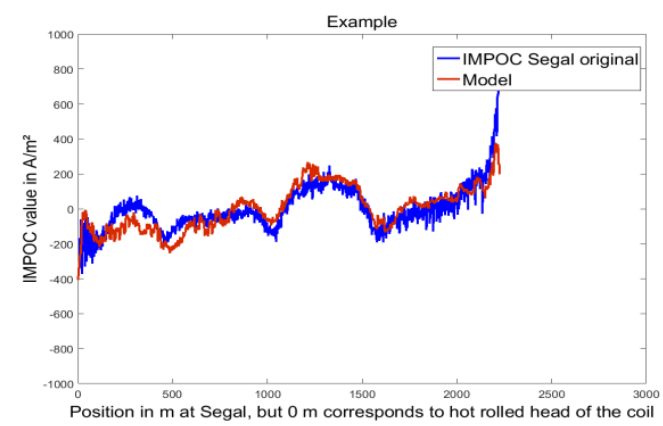

Fig.10 - Comparison of modeled (red) and real measured (blue) development of the tensile strength within a DP 800 coil

\section{BIG RIVER STEEL - APPLICATION EXAMPLE}

\subsection{Universal hot-dip galvanizing and annealing line with I-Furnace}

SMS group delivers a universal annealing and galvanizing line for cold-strip treatment at Big River Steel's new mill complex in Osceola, Arkansas, USA. One of the unique features of the line is its innovative design because it allows for the production of two different products (annealed or galvanized) and thus makes it possible to respond to changes in the market and accomplish maximum cost efficiency.

The new line will enable Big River Steel to produce steel grades from commercial to automotive such as CQ, DQ, DDQ and IF as well as high-strength steel grades like HSLA, DP and CP. The total annual capacity for galvanized and annealed material will be approximately 476,500 tons. Strip will be produced in thicknesses from 0.36 to 3.0 millimeters and widths from 900 to 1,850 millimeters at a process speed of up to 150 meters per minute. At the entry and exit sections, speeds of up to 220 meters per minute can be achieved. SMS has designed the mechanical equipment, manufactured special components, and supplied the complete electrical and automation systems for the line.

The line will be equipped with a Drever International annealing furnace. The strip is heated in the furnace by direct flame firing and radiant tube burners. Of particular note is the ultra-fast cooling system which has the capacity to cool the strip at very high cooling rates so that the plant can produce high-strength automotive steel grades having less costly alloying elements. Furthermore, the furnace is recognized as eco-friendly with several energy recovery features and NOx treatment equipment in the exhaust system.

The line will feature the I-Furnace system. Therefore, an advanced mathematical/physical model will control the furnace. The EMG IMPOC system will be integrated behind the tension leveler of the line to measure the material strength of the processed strips. Last but not least the data driven Annealing Microstructure Model will be implemented to predict the material characteristics.

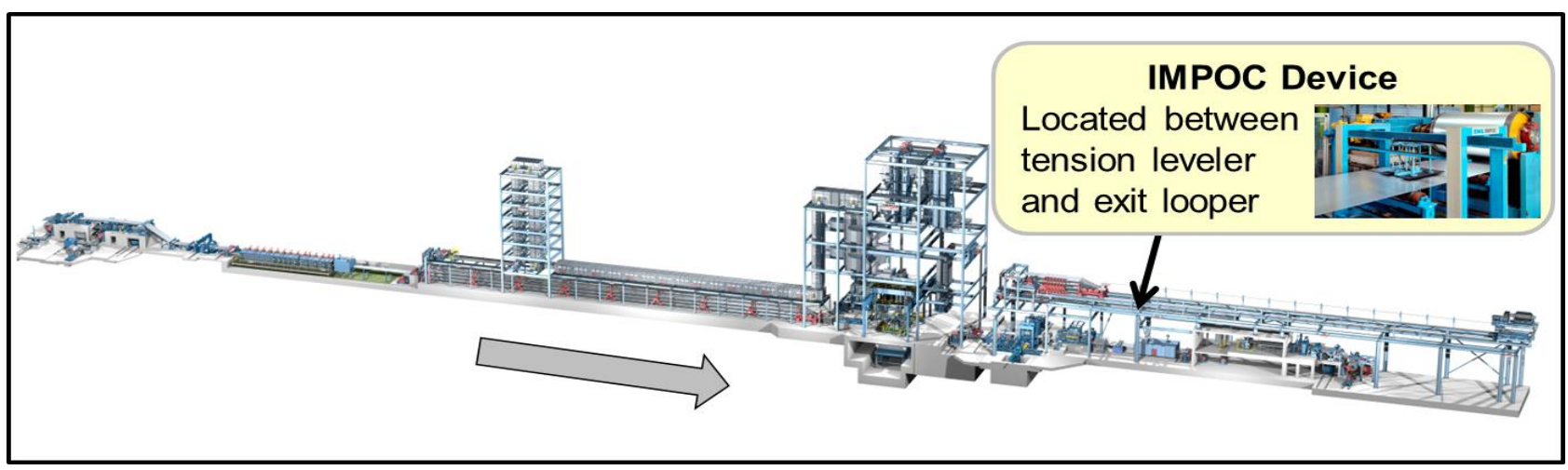

Fig.11 - New universal annealing and galvanizing line for Big River Steel with integrated EMG IMPOC system 


\subsection{Implementation steps}

The I-Furnace in the Big River Steel annealing and galvanizing line will be implemented within three subsequent steps. With each implementation step the benefits for the producer are increasing.

The implementation will start with the monitoring phase. During commissioning relevant data will be validated. Results from destructive tests of material properties will be collected in order to do the regression for the EMG IMPOC device. When this phase is completed, the learning phase starts. This comprises of an offline data analysis in order to predict the mechanical properties for each steel type and from coil to coil. As soon as this learning phase is completed, improving of the operation will start. The online model will allow automatic changes of the furnace setup, which will result in higher yield and less energy consumption.

\section{CONCLUSION}

The newly developed I-Furnace ensures exact heat treatment processes for an efficient achievement of defined material properties. Thus, it is considered to be the most advanced system for the economic and environmental friendly production of modern steel grades. The optimizations of the quality and the increased production at Segal's galvanizing line have demonstrated the capability of the system. The application of the I-Furnace at Big River Steel's new universal annealing and galvanizing line will once again show the potential of this system for modern cold-strip processing lines.

\section{REFERENCES}

